\title{
Reacción anafiláctica al látex durante cirugía
}

\author{
Anaphylactic reaction to latex during surgery \\ Melissa Linares Hernández,* Francisco Ramírez Bernal," \\ Fabio Andrés Avellaneda Suárez, ${ }^{\S}$ Rocío Areli Rojas Jaimes
}

Citar como: Linares HM, Ramírez BF, Avellaneda SFA, Rojas JRA. Reacción anafiláctica al látex durante cirugía. Acta Med. 2021; 19 (1): 116-122. https://dx.doi.org/10.35366/98580

\section{Resumen}

Los productos que contienen látex natural son causa de reacciones anafilácticas graves durante cirugía. El anestesiólogo debe ser consciente del riesgo potencial de un evento de choque anafiláctico. El siguiente artículo describe el caso de una mujer de 41 años programada para la resección de un tumor pancreático distal. La paciente se conocía alérgica a dicho material. Antes de su ingreso a quirófano se brindó premedicación. Durante el evento quirúrgico desarrolló hipotensión severa, taquicardia y broncoespasmo; se documentó contacto accidental con sonda de látex para drenaje tipo Penrose. Se dieron medidas de soporte, logrando estabilización hemodinámica de la paciente. Se dio manejo postquirúrgico en la Unidad de Terapia Intensiva. Los productos de látex natural pueden ser causa de reacciones anafilácticas transoperatorias, incluyendo el choque anafiláctico. El diagnóstico muchas veces se ve retrasado por las complicaciones propias de las técnicas anestésicas y quirúrgicas (hipotensión, broncoespasmo). El anestesiólogo debe mantener en mente un evento de choque anafiláctico como causa de hipotensión refractaria a tratamiento convencional, de este modo, se logrará instaurar de manera oportuna el tratamiento pertinente.

Palabras clave: Látex, anafilaxia, choque anafiláctico, complicaciones perioperatorias, anestesia.

\section{Abstract}

Products containing natural latex are the cause of severe anaphylactic reactions during surgery. The anesthesiologist must be aware of the potential risk of an anaphylactic shock event. The following article describes the case of a 41-yearold woman scheduled for the resection of a distal pancreatic tumor. The patient was known to be allergic to latex. Prior to entering the operating room, premedication was provided. During the surgical event, severe hypotension, tachycardia and bronchospasm developed; accidental contact with a latex probe for Penrose drainage was documented. Support measures were given, achieving hemodynamic stabilization of the patient. Postoperative management was provided in the Intensive Care Unit. Natural latex products may be the cause of transoperative anaphylactic reactions, including anaphylactic shock. The diagnosis is often delayed due to the complications of anesthetic and surgical techniques (hypotension, bronchospasm). The anesthesiologist must keep in mind an anaphylactic shock event as a cause of hypotension refractory to conventional treatment, in this way, the appropriate treatment will be established in a timely manner.

Keywords: Latex, anaphylaxis, anaphylactic shock, perioperative complications, anesthesia.

$1: 3,500$ a $1: 20,000$ y muchos anestesiólogos no tienen la experiencia de haberse enfrentado a un episodio de anafilaxia. ${ }^{2}$ Es fundamental identificar la reacción anafiláctica para hacer un diagnóstico precoz y establecer un pronto plan terapéutico, siguiendo un algoritmo de
Es importante concientizar al anestesiólogo sobre el riesgo de anafilaxia durante la anestesia. ${ }^{1}$ La incidencia de un evento de anafilaxia transanestésico varía de
* Médica Anestesióloga.

₹ Médico Anestesiólogo y Residente de segundo año de Medicina Crítica.

$\S$ Residente de tercer año de Anestesiología.

" Médica adscrita Unidad de Anestesiología, Anestesiología Cardiovascular.

Centro Médico Nacional "20 de Noviembre, ISSSTE".
Correspondencia:

Dra. Rocío Areli Rojas Jaimes

Correo electrónico: rareli@prodigy.net.mx

Aceptado: 07-09-2020.

www.medigraphic.com/actamedica

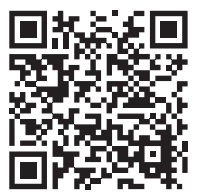


actuación bien establecido. ${ }^{1}$ Dentro del quirófano, los elementos más frecuentemente implicados en las reacciones de anafilaxia perioperatoria son: los bloqueadores neuromusculares (60\%), el látex (15\%), y los antibióticos $(15 \%) .^{1}$

Anafilaxia se define como una reacción alérgica grave, de inicio brusco, que afecta múltiples órganos y sistemas, y que pone en peligro la vida del paciente; ${ }^{1}$ se puede clasificar en dos.

El término anafilaxia alérgica o inmunomediada debe utilizarse cuando en la reacción anafiláctica está implicado un mecanismo inmunológico, normalmente mediado por anticuerpos IgE (60\%) o IgG. ${ }^{1}$ Este tipo de anafilaxia es un síndrome sistémico de hipersensibilidad inmediata que corresponde al tipo I de acuerdo con la clasificación de Gell y Coombs, y causa la liberación de mediadores preformados de mastocitos y basófilos. Este tipo de reacción requiere de un proceso de sensibilización llamado "primer contacto" con el alérgeno o antígeno y la formación consecuente de un anticuerpo específico. Durante el primer contacto no se desencadena la reacción anafiláctica. ${ }^{2}$

El término anafilaxia no alérgica o no inmunomediada describe la misma clínica, pero el mecanismo no está mediado por anticuerpos, es el resultado de la liberación directa y no especifica de mediadores vasoactivos y proinflamatorios al torrente circulatorio. ${ }^{1}$

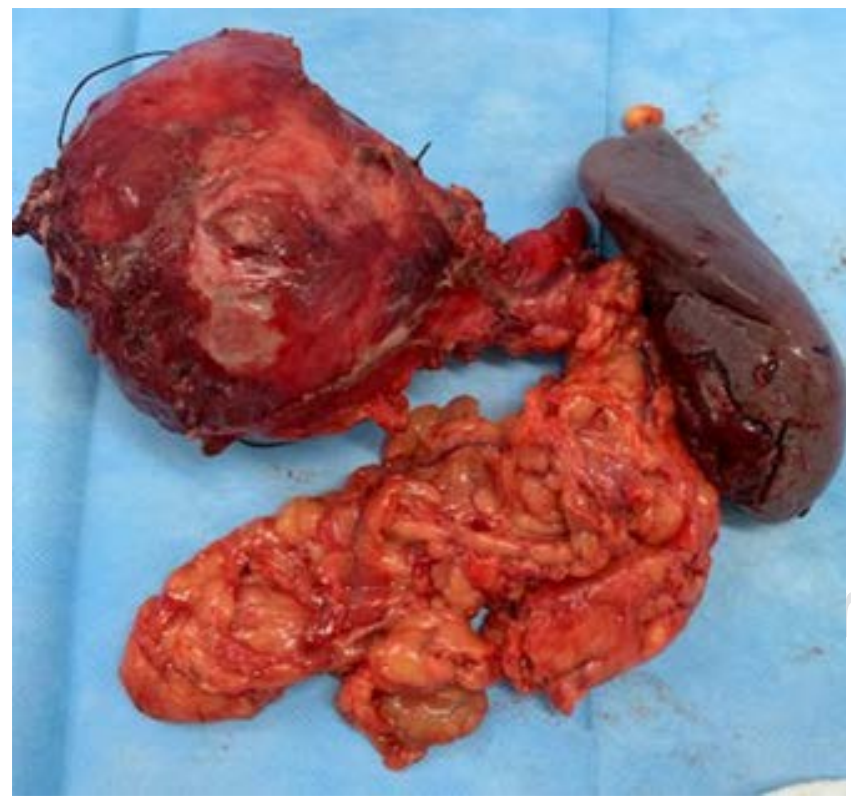

Figura 1: Pieza quirúrgica producto de la resección. Se observa páncreas distal con presencia de una tumoración en uno de sus extremos, así como un fragmento de hígado.
El objetivo de este reporte de caso es conocer e identificar una reacción alérgica al látex, en los pacientes sometidos a intervención quirúrgica, y de esta manera actuar de forma oportuna y rápida al presentarse durante el transanestésico.

\section{PRESENTACIÓN DE CASO}

Se trata de un paciente femenino de 41 años, con diagnóstico preoperatorio de tumor pancreático distal. Ingresa a quirófano para la realización de pancreatectomía distal. Dentro de los antecedentes de importancia destacan ERGE, hipotiroidismo, ambos en tratamiento, así como asma bronquial en tratamiento con salmeterol y fluticasona, última crisis documentada un año previo a la fecha de programación de la cirugía. Se realizó diagnóstico de quiste pancreático en abril 2018. Dentro de los antecedentes alérgicos manifiesta alergia a la papaya y al aguacate, los cuales le producen rash cutáneo y angioedema; además de alergia al látex, manejado con vacunas por Servicio de Alergología (última vacuna hace dos meses). Quirúrgicos: dos cesáreas e histerectomía por miomatosis uterina manejadas con anestesia neuroaxial; además de colocación bajo sedación de prótesis endoscópica en conducto pancreático principal.

Se programa como la primera cirugía del turno. Previo a ingresar a quirófano, se premedica con hidrocortisona $100 \mathrm{mg}$ IV, difenhidramina $30 \mathrm{mg}$ IV y ranitidina $50 \mathrm{mg} \mathrm{IV}$. Ingresa a sala de quirófano, consciente, neurológicamente íntegra, con signos iniciales: TA 135/80, TAM 103 mmHg, FC 85 lpm, SpO 2 90\%, FR 12 rpm. Se administra ansiólisis con midazolam $2 \mathrm{mg} I \mathrm{~V}$, se realizó inducción anestésica con fentanilo $200 \mu \mathrm{g}$ IV, propofol $150 \mathrm{mg}$ IV, cisatracurio $10 \mathrm{mg}$ IV. Previa preoxigenación con mascara facial a 5 Ipm y latencia farmacológica, se orointuba con sonda orotraqueal $\mathrm{N}^{0} 7.5$ al primer intento sin complicaciones, y se conecta a ventilador mecánico en modo controlado por volumen. Se toma línea arterial radial izquierda. El mantenimiento con infusión de fentanilo a $3 \mu \mathrm{g} / \mathrm{kg} / \mathrm{hora}$, sevoflurano 1.5 a 2 vol. \%, en adecuado plano anestésico. Una vez realizada la resección (Figura 1), transcurridas tres horas de cirugía, se procedió al cierre y colocación de drenajes. La paciente presenta desaturación gradual llegando a $60 \%$, además de hipotensión hasta 50/20 $\mathrm{mmHg}$ con TAM de $30 \mathrm{mmHg}$. Se inició reanimación hídrica con cristaloides y coloides sin respuesta adecuada, por lo que se administraron 2 gramos de gluconato de calcio IV. Se presentó además broncoespasmo severo, por lo que se inició ventilación manual a presión positiva y se administraron cuatro disparos de salbutamol por cánula orotraqueal. Al no mejorar la hipotensión, se suspendió la infusión de fentanilo, se procedió a verificar posibles 
Figura 2:

Signos vitales, presión arterial y frecuencia cardiaca en el transanestésico.
120

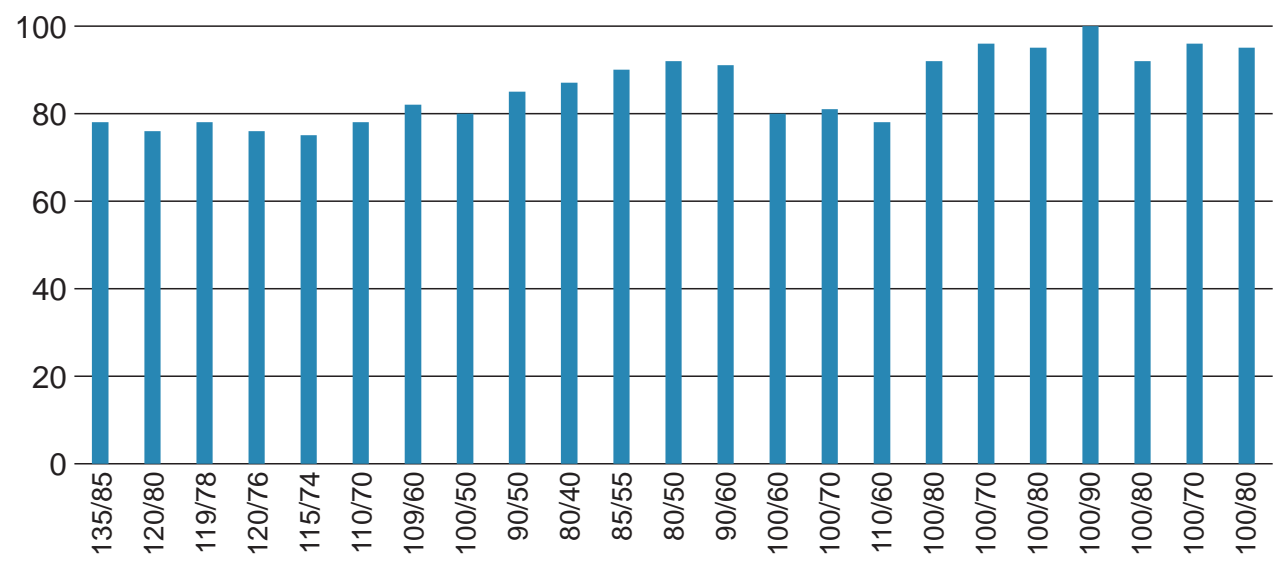

Fuente: Los autores. sitios de sangrado sin hallarse, se decidió iniciar infusión de noradrenalina a $0.03 \mu \mathrm{g} / \mathrm{kg} / \mathrm{min}$. Del mismo modo, el broncoespasmo no presentaba mejoría por lo que se administraron tres disparos de bromuro de ipratropio/ salbutamol por cánula orotraqueal y $250 \mathrm{mg}$ de aminofilina IV, incluso se administra adrenalina, dosis de $80 \mu \mathrm{g}$ a través del tubo orotraqueal, con lo que finalmente se logró reversión. Se evidencia en cavidad abdominal sonda de Penrose (único contacto con látex durante todo el procedimiento), se procede a su retiro. Se aplican $0.7 \mu \mathrm{g}$ de adrenalina IM, y se inicia infusión de ésta a $0.03 \mu \mathrm{g} /$ $\mathrm{kg} / \mathrm{min}$, se aumentó infusión de noradrenalina de forma gradual hasta una dosis máxima de $0.10 \mu \mathrm{g} / \mathrm{kg} / \mathrm{min}$ con lo que mejora la tensión arterial. Termina procedimiento quirúrgico. La paciente se traslada a unidad de terapia intensiva con TA de 100/70 mmHg, TAM $80 \mathrm{mmHg}$, $\mathrm{SpO}_{2}$ 95\%, FC 95 lpm (Figura 2) en ventilación asistida, con infusión de fentanilo a $1 \mu \mathrm{g} / \mathrm{kg} / \mathrm{h}$, noradrenalina a $0.5 \mu \mathrm{g} / \mathrm{kg} / \mathrm{min}$ y adrenalina a $0.03 \mu \mathrm{g} / \mathrm{kg} / \mathrm{min}$. En la unidad de terapia intensiva mantiene adecuada evolución posquirúrgica logrando su alta hospitalaria a los 20 días postquirúrgicos.

\section{DISCUSIÓN}

El primer informe sobre urticaria asociada con caucho de látex se describió en 1927 en Alemania por Grete Stern, un caso típico de alergia a este caucho por hipersensibilidad tipo 1.3,4 Los primeros reportes durante actos quirúrgicos datan de 1987, en ese momento, la alergia al látex representaba sólo el $10 \%$ de los eventos anafilácticos transquirúrgicos, dado que la anafilaxia por fármacos es más común y el látex no era un común denominador en ese tiempo, por lo que incluso muchos eventos pasaron desapercibidos. $^{5}$

El látex se describe como un fluido lechoso obtenido de los vasos laticíferos del árbol del caucho, Hevea brasiliensis. Está compuesto principalmente por cis-1,4-polisopreno, un polímero orgánico que le confiere su fuerza y elasticidad, además de más de 200 polipéptidos, cada uno con un potencial alergénico variable. ${ }^{6}$

Entre los muchos componentes del látex, la proteína heveina es el antígeno responsable de la anafilaxia. Existen quince proteínas alergénicas probadas, identificadas como Hevb1 hasta la Hevb15, las cuales pueden provocar una respuesta inmune hipersensible. ${ }^{7}$ Los pacientes con alergia a este material tienen una alta tasa de sensibilización a Hevb5 conocida como "proteína de látex ácida", a Hevb6.01 "proheveina" y a Hevb6.02 "heveina". Los trabajadores de la salud que presentan alergia al látex, debido a la sensibilización cutánea a través de los guantes, muestran altas tasas de sensibilización a Hevb5, Hevb6.02 ${ }^{8}$ y Hevb7. ${ }^{9}$ La proteína Hevb1 "factor de elongación del caucho" y Hevb3 "proteína de partículas pequeñas de caucho", se consideran alérgenos muy importantes entre los niños con espina bífida ${ }^{8}$ o que cuentan con antecedente de una deformidad urológica congénita.

En las décadas de 1980 y 1990 el mayor uso de guantes para prevenir el contacto con nuevas enfermedades, además del uso del látex en otros objetos produjo un aumento de la demanda de éste, favoreciendo la aparición 
de cientos de nuevas, y a veces poco reguladas, fábricas. De forma paralela, comenzó a aumentar rápidamente la incidencia de reacciones alérgicas leves y graves al látex entre los pacientes y los profesionales sanitarios. ${ }^{6}$ Hoy en día, este elemento está presente en muchos productos alimenticios y médicos. Incluso la Administración de Drogas y Alimentos de los EUA (FDA) ha aprobado la aplicación de látex en aditivos alimentarios (goma de mascar), envases de alimentos (adhesivos, recubrimientos, antioxidantes) y dispositivos médicos (dentales, de uso en gastroenterología y cirugía). ${ }^{7}$

La sensibilización al látex puede ocurrir tras el contacto con la piel o las mucosas, tras el contacto con el peritoneo durante la cirugía, y posiblemente tras inhalación de partículas en aerosol con látex en sus superficies. ${ }^{6}$ Como se ha mencionado, muchos artículos de uso médico común contienen partículas de látex en sus superficies (guantes, sondas, catéteres, tapones de viales), además, los antígenos del látex pueden ser inhalados en forma de las partículas volátiles provenientes de guantes de látex o de polvos industriales utilizados en la fabricación de neumáticos. ${ }^{7}$

La prevalencia promedio de alergia al látex en la población general en todo el mundo es de $4.3 \%{ }^{7}$ Es más frecuente en grupos de población con exposición laboral. ${ }^{6}$ La prevalencia actual de alergia y sensibilización al látex, entre los trabajadores de la salud en todo el mundo, es del 9.7 y $12.4 \%$, respectivamente. ${ }^{7}$ La prevalencia de la alergia oscila en $10 \%$ en los trabajadores de la industria del caucho. ${ }^{6}$ La mayor prevalencia de alergia al látex se encuentra en pacientes con espina bífida o anomalías congénitas genitourinarias, con una prevalencia actual de alergia y sensibilización de 7.2 y $30.4 \%$, respectivamente. ${ }^{6,7}$ La sensibilización en estos pacientes aparentemente se debe a instrumentación frecuente sobre el aparato urinario, la zona rectal y el espacio dural, así como a diversas cirugías durante la primera infancia, se ha sugerido incluso que pueden tener una predisposición genética para la sensibilización al látex. ${ }^{6}$ Por último, se ha visto una mayor prevalencia de alergia al látex en personas con alergias a frutas y semillas (aguacate, kiwi, papaya, melocotón, nectarina, plátano, pera, guayaba, fresa, uva, higo, mango, frutos secos, castaña, coco, cacahuate), carnes (pescado), verduras, legumbres y hierbas de olor (zanahoria, pimiento, orégano, salvia, espinaca, alubias, betabel, papa, tomate). ${ }^{6}$ Los pacientes con alergia a estas frutas tienen un alto riesgo de reactividad cruzada y desarrollan una alergia conocida con el nombre de "síndrome del látex-fruta"; cuando entran en contacto con productos derivados del látex, de hecho, aproximadamente entre el 30 a 50\% de las personas con alergia al látex muestran una hipersen- sibilidad asociada a uno o más de estos elementos. ${ }^{7}$ La proteína Hevb 6.02 se conoce como componente causal importante en el síndrome "látex-fruta", esto debido a la alta similitud de la secuencia de aminoácidos de ésta y la quitinasa clase 1 contenida en bananos, aguacates, kiwis, castaños, etcétera. ${ }^{8}$ La paciente que se aborda en el caso clínico aquí presentado presentaba alergia a papaya y aguacate.

El caucho de látex natural causa dos tipos diferentes de reacciones alérgicas; la no inmunomediada, y la reacción de tipo I inmediata o inmunomediada. ${ }^{4}$

Los mediadores responsables de las reacciones alérgicas son: ${ }^{2}$

- Mediadores preformados: histamina, proteasas, triptasa, quimasa, heparina, factor liberador de histamina y otras citocinas.

- Mediadores neoformados: leucotrienos, prostaglandinas, factor activador de plaquetas.

La histamina es la principal molécula liberada en las reacciones alérgicas y es responsable de una gran parte de la sintomatología observada, debido al efecto sobre receptores $\mathrm{H} 1$ y $\mathrm{H} 2$ presentes esencialmente en los órganos blanco de la reacción alérgica. Síntomas como el prurito, la rinorrea, taquicardia y broncoespasmo están causados por la activación de receptores H1, además, la histamina unida a los receptores $\mathrm{H} 1$ estimula a las células endoteliales para convertir la L-arginina en óxido nítrico, un potente vasodilatador. ${ }^{2}$ Por otra parte, los leucotrienos y el ácido 5-HETE son productos de la vía de la lipooxigenasa que toma como sustrato el ácido araquidónico de las membranas celulares. El LTB4 tiene actividad quimiotáctica sobre polimorfonucleares y es un agregante plaquetario, LTC4, LTD4 y LTE4 son broncoconstrictores muy importantes, mil veces más potentes que la histamina. ${ }^{2}$ Las prostaglandinas provienen de la acción de la ciclooxigenasa sobre el ácido araquidónico, estos mediadores modulan la contractilidad del músculo liso y aumentan la permeabilidad capilar. ${ }^{2}$ Finalmente, el factor activador de plaquetas es un derivado directo de los fosfolípidos de las membranas mastocitarias y provoca un aumento de la permeabilidad capilar siendo un potente inductor de choque. ${ }^{2}$

Sugimoto informó que la reacción alérgica típica al látex ocurre dentro de los 30 a 60 minutos después de la exposición. ${ }^{8}$ Según el patrón de presentación temporal, el caso de anafilaxia presentado corresponde al del tipo unifásico. Se distinguen, según esta característica, tres tipos de temporalidad, en primer término, la anafilaxia unifásica, que es la más frecuente, caracterizada por un pico sintomático clásico que se resuelve espontáneamen- 
te o con tratamiento en los 30 a 60 min siguientes; en segundo término, se describe la anafilaxia bifásica, en la cual, tras una primera reacción, aparece un periodo asintomático entre una a ocho horas, seguido de otro pico sintomático sin haber sufrido una segunda exposición al alérgeno implicado; por último, la anafilaxia prolongada que tiene una duración mayor de 24 horas de la sintomatología. ${ }^{10}$ Ya que la sintomatología puede reaparecer con intensidad variable y no predecible, se recomienda tener bajo vigilancia al paciente una vez que se haya presentado un evento, ${ }^{1}$ esta última razón es la justificación de que los pacientes que sufren un evento de anafilaxia sean vigilados según el grado de intensidad en la unidad de recuperación postanestésica, piso de hospitalización o incluso en la Unidad de Cuidados Intensivos como en el caso de esta paciente.

Las manifestaciones clínicas de la anafilaxia pueden ser muy variadas, debiendo considerarse diversos diagnósticos diferenciales. ${ }^{1}$ En la Tabla 1 se listan los posibles signos y síntomas de anafilaxia.

Durante la anestesia, los síntomas cardiovasculares, cutáneos, y el broncoespasmo son los signos clínicos más frecuentes. Las manifestaciones cutáneas son difíciles de identificar de manera inicial, porque el paciente generalmente se encuentra cubierto con campos durante el procedimiento quirúrgico. Los signos respiratorios están minimizados por las propiedades broncodilatadoras de los anestésicos inhalados y la hipotensión farmacológica es

Tabla 1: Signos y síntomas de la anafilaxia. ${ }^{10}$

\begin{tabular}{|c|c|}
\hline Sistema & Síntomas \\
\hline Piel & $\begin{array}{l}\text { Enrojecimiento de la piel; urticaria; } \\
\text { inflamación de la cara, los labios o la } \\
\text { lengua; prurito }\end{array}$ \\
\hline Respiratorio & $\begin{array}{l}\text { Aumento de la frecuencia respiratoria, } \\
\text { estridor, sibilancias, disminución de } \\
\text { la saturación de oxígeno, cianosis, } \\
\text { congestión nasal, rinorrea, estornudos }\end{array}$ \\
\hline Cardiovascular & $\begin{array}{l}\text { Taquicardia, hipotensión, dolor en el } \\
\text { pecho, choque, paro cardiaco }\end{array}$ \\
\hline Gastrointestinal & $\begin{array}{l}\text { Náuseas, dolor abdominal, vómitos, } \\
\text { diarrea }\end{array}$ \\
\hline Neurológico & $\begin{array}{l}\text { Ansiedad, dolor de cabeza, mareo, } \\
\text { confusión, sentimiento de muerte } \\
\text { inminente }\end{array}$ \\
\hline
\end{tabular}

Los síntomas de anafilaxia pueden englobar toda la economía. Dentro de los síntomas más graves están los asociados al sistema cardiovascular y respiratorio.
Tabla 2: Clasificación de la gravedad de las reacciones de anafilaxia durante la anestesia. ${ }^{1}$

\section{Grado Manifestaciones clínicas}

I Signos cutáneos generalizados: eritema,

urticaria con o sin angioedema

II Afectación multiorgánica moderada con signos cutáneos, hipotensión y taquicardia, hiperreactividad bronquial

III Afectación multiorgánica grave que requiere tratamiento específico vital: colapso, taquicardia, bradicardia, arritmias, broncoespasmo

IV Paro respiratorio o cardiaco

$\mathrm{V} \quad$ Muerte debida a la falta de respuesta a la reanimación cardiopulmonar

De acuerdo con los sistemas involucrados y la gravedad de los síntomas se establecerá la gravedad de la reacción anafiláctica, y de ello también dependerá el tratamiento.

común, es por esto por lo que el diagnóstico y tratamiento inicial se retrasa con frecuencia. ${ }^{2}$ Ciertamente, durante la cirugía, la primera sospecha de colapso cardiovascular o respiratorio no es la anafilaxia; las complicaciones más frecuentes inherentes a la propia técnica anestésica, por ejemplo bloqueo simpático extenso en la anestesia regional o el broncoespasmo en anestesia general, forman parte del diagnóstico diferencial y acaban apareciendo primero como opción diagnóstica.

En cuanto a gravedad, las reacciones de anafilaxia de tipo inmunomediada son las más graves, causando colapso cardiovascular en un 50\% de las ocasiones. ${ }^{1}$ De acuerdo con la gravedad de la clínica, se ha establecido una clasificación de reacciones de anafilaxia, la cual se reporta en la Tabla 2.

El tratamiento de una reacción anafiláctica transoperatoria se basa en tres pilares: medidas generales, tratamiento de primera línea y tratamiento de segunda línea. ${ }^{1}$

Medidas generales. Constituyen las siguientes: suspensión de la administración o contacto con la sustancia sospechosa, realizar valoración de aparatos y sistemas (vía aérea, respiratorio, circulatorio), informar al equipo quirúrgico de la situación y solicitar ayuda, suspender o acortar la intervención quirúrgica, administrar oxígeno al $100 \%$, colocar al paciente en posición de Trendelenburg. ${ }^{1}$

Primera línea de tratamiento. Consiste en la administración de adrenalina y fluidoterapia.

Administración de adrenalina. Es el fármaco de primera elección en caso de anafilaxia y debe ser administrado 
lo antes posible. El efecto alfa adrenérgico produce vasoconstricción, que reduce la vasodilatación periférica, mejora la hipotensión, el eritema, la urticaria y el angioedema. El efecto beta adrenérgico provoca broncodilatación, aumenta el gasto cardiaco y la contractilidad, además de impedir la liberación de mediadores de los mastocitos y basófilos. Sin embargo, en casos como el asma o la medicación con beta bloqueadores su administración no suele ser efectiva. En caso de reacción grado II 0.01 $\mathrm{mg} / \mathrm{kg} \mathrm{IM}$, máximo $0.5 \mathrm{mg}$ y repetir cada 5 a 15 minutos de ser necesario. En caso de reacción grado III $50 \mu \mathrm{g}$ IV, de ser necesario iniciar perfusión. En caso de reacción grado IV se puede llegar hasta $1 \mathrm{mg}$ por minuto e iniciar perfusión de 4-10 $\mu \mathrm{g} / \mathrm{min})$.

Fluidoterapia. Durante un evento de anafilaxia, el líquido intravascular se extravasa al espacio intersticial hasta en un $50 \%$ en 10 minutos. Es fundamental la administración de fluidos. Se recomienda reemplazo de volumen a $20 \mathrm{~mL} / \mathrm{kg}$ dentro de los primeros 5 a 10 minutos, ${ }^{1,4}$ llegando hasta $50 \mathrm{~mL} / \mathrm{kg}$ en la primera hora si es necesario.

La segunda línea de tratamiento consiste en la administración de glucocorticoides, antihistamínicos, y beta agonistas. $^{1}$

Glucocorticoides. Impiden la transcripción de los genes que codifican las proteínas proinflamatorias, aunque su inicio es tardío. Pueden aliviar los síntomas de larga duración y prevenir los episodios de anafilaxia bifásica. Se recomienda hidrocortisona de 100 a 200 mg IV o metilprednisolona de 50 a 100 mg IV. ${ }^{1}$

Antihistamínicos H1. Alivian el rubor, la urticaria, el prurito y el angioedema, pero no mejoran los síntomas que pueden llegar a provocar la muerte. Se puede usar difenhidramina 25 a $50 \mathrm{mg}$ IV o clorfenamina 5 a $10 \mathrm{mg} \mathrm{IV.}{ }^{1}$

Antihistamínicos $\mathrm{H} 2$. Junto a los anti $\mathrm{H} 1$ pueden contribuir a disminuir los síntomas. Un ejemplo es la ranitidina, en una dosis de $50 \mathrm{mg} \mathrm{IV.}{ }^{1}$

Agonistas B2. Fármacos como el salbutamol pueden ser útiles en la anafilaxia cuando hay broncoespasmo que no resuelve con la adrenalina. No sustituyen a la adrenalina ya que sólo son útiles a nivel ventilatorio. Se puede utilizar en forma de inhalador dosis medida o en forma IV a una dosis de 100 a $200 \mu$ g con perfusión de 5 a $25 \mu \mathrm{g} / \mathrm{min}^{1}{ }^{1}$

Las pruebas aprobadas de confirmación para la alergia al látex consisten en análisis de sangre que evalúan la IgE antilátex. Estas pruebas tienen una especificidad relativamente alta, pero sólo debe interpretarse en el contexto de la historia de las exposiciones y reacciones del paciente. ${ }^{10}$

En el caso de las cirugías de urgencia, la anestesia se realiza muchas veces por el anestesiólogo en su primer contacto con el paciente en el quirófano. Este no es el ambiente ideal para una investigación clínica. El anestesiólogo debe ser lo más claro posible al interrogar a los pacientes, ya que los datos de ingreso a menudo son insuficientes para proporcionar información fundamental sobre las comorbilidades del paciente. ${ }^{3}$

El tratamiento preoperatorio de una alergia al látex o una sospecha de alergia al látex implica difenhidramina (1 mg/ $\mathrm{kg}$ cuatro veces al día), ranitidina (2 $\mathrm{mg} / \mathrm{kg}$ tres veces al día) e hidrocortisona ( $5 \mathrm{mg} / \mathrm{kg}$ cuatro veces al día) administradas desde una hora antes de la cirugía a un día después de la operación. ${ }^{9}$ En este caso, se procedió con la premedicación del paciente de esta forma.

La mejor técnica de manejo es la prevención, y la mejor medida preventiva es la evitación. Más importante aún, los pacientes deben ser conscientes de su condición, para que puedan evitar los productos de látex. También deben informar a los que los rodean de su alergia, para casos de emergencia. ${ }^{6}$

En 1997, la FDA comenzó a exigir el etiquetado en todos los equipos médicos que contienen látex. Muchos productos combinados que contienen látex no reportan contener látex en sí, por ejemplo, los tapones de goma para viales de medicamentos, en ese caso, para disminuir el riesgo, se recomienda seleccionar viales de un solo uso o viales de uso múltiple nuevos (nunca perforados anteriormente) al dispensar medicamentos a pacientes alérgicos. Otros productos que pueden contener látex, como los manguitos de presión arterial y los tubos del estetoscopio, se pueden cubrir con un paño para mantener un entorno seguro para el paciente. ${ }^{10}$

Otro paso útil es programar el procedimiento del paciente como el primer caso del día, después de que la sala se haya limpiado a fondo y antes de que exista una posible contaminación por productos de látex durante el transcurso del día. ${ }^{10}$

\section{REFERENCIAS}

1. Escolano VF, Sánchez NS. Anafilaxia en anestesia. Rev Esp Anestesiol Reanim. 2013; 60 (1): 55-64.

2. Rivera-Ramírez OA, Mendoza-Magaña ME, Racette M. Anestesia y anafilaxia. Rev Mex Anestesiol. 2008; 31 (1): 37-44.

3. Machado JA, Da Cunha, Romilton Crozetta, De Oliveira, Benhur Heleno, Da Silva, Jane. Latex-induced anaphylactic reaction in a patient undergoing open appendectomy. Case report. Rev Bras Anestesiol. 2011; 61 (3): 360-366.

4. Malsy M, Leberle R, Ehehalt K, Sinner B, Hobbhahn J. Anaphylactic reaction 5 minutes after the start of surgery: a case report. BMC Res Notes. 2015; 8 (117): 1-4.

5. Leynadier F, Pecquet C, Dry J. Anaphylaxis to latex during surgery. Anaesthesia. 1989; 44 (7): 547-550.

6. Huerta López, José. Alergia al látex. Alerg Asma Inmunol Pediatr. 2008; 17 (2): 65-69. 
7. Wu Miaozong, Mclntosh J, Liu J. Current prevalence rate of latex allergy: why it remains a problem? J Occup Health. 2016; 58 (2): 38-144.

8. Sugimoto $M$, Ono A, Yada K, Mori H, Ishibashi H, Niki N, Kagami S. Manifestation of intraoperative anaphylactic shock along with latex allergy: a pediatric case report. J Med Invest. 2018; 65 (3.4): 292-295.
9. Lee W, Lee JH, Park DJ, Kim HH. A case of anaphylactic shock attributed to latex allergy during gastric cancer surgery. J Korean Surg Soc. 2011; 81 (Suppl. 1): S30-33.

10. Martínez-Fariñas P, González-Arévalo A, Martínez-Hurtado ED, Chacón M, García del Valle S. Shock anafiláctico prolongado de 4 días de duración. Rev Esp Anestesiol. 2013. Disponible en: http:// dx.doi.org/10.1016/j.redar.2013.09.017. 